there was no significant difference in mortality rates between those with and without MV CAD (HR 1.34 [95\% CI 0.971.85], $\mathrm{p}=0.07)$. (Figure 1A). There was also no significant difference in mortality in patients with MV CAD greater than 80 years old undergoing CORV versus FRV $(p=0.19)$. (Figure 2).

Conclusions The results show there is a mortality difference in patients with MV versus SV CAD in patients less than 80 years old, which is not seen in patients greater than 80 years old. There also does not appear to be a mortality benefit in FRV compared with CORV in the elderly. While a reduction in cardiovascular mortality with FRV has been demonstrated in a recent meta-analysis these results indicate that this benefit may be tempered in elderly patients. This is a single centre study with relatively small numbers, especially in those greater than 80 years old undergoing FRV. Given the uncertainty around FRV in the elderly, there remains a need for a randomised control trial to evaluate this question.

Conflict of Interest None

\section{DRUG COATED BALLOON ONLY ANGIOPLASTY FOR STEMI}

${ }^{1}$ Ioannis Merinopoulos, ${ }^{2}$ Tharusha Gunawardena, ${ }^{2}$ Natasha Corballis, ${ }^{3}$ Upul Wickramarachchi, ${ }^{3}$ Clint Maart, ${ }^{3}$ Sulfi Sreekumar, ${ }^{3}$ Chris Sawh, ${ }^{3}$ Trevor Wistow, ${ }^{3}$ Toomas Sarev, ${ }^{3}$ Tim Gilbert, ${ }^{3}$ Alisdair Ryding, ${ }^{4}$ Vassilios Vassiliou, ${ }^{3}$ Simon Eccleshall. ${ }^{1}$ University of East Anglia, Norfolk \& Norwich University Hospital, Norwich, UK; ${ }^{2}$ Norwich Medical School and Norfolk \& Norwich University Hospital; ${ }^{3}$ Norfolk \& Norwich University Hospital; ${ }^{4}$ Norwich Medical School, University of East Anglia

\subsection{6/heartjnl-2021-BCS.48}

Introduction In the setting of primary percutaneous coronary intervention (PPCI), it has been shown that stenting compared to balloon angioplasty (BA) reduces the need for repeat revascularisation but not overall mortality. A recent, small randomised trial showed that drug coated balloon (DCB) angioplasty for PPCI was non-inferior to drug eluting stent (DES) in terms of fractional flow reserve at 9 months. Our aim was to investigate the safety of DCB angioplasty in a STEMI population in terms of all-cause mortality.

Methods We identified all patients treated for STEMI in our institution from January 2016 until November 2019. We excluded patients who died in hospital and patients with cardiac arrest, cardiogenic shock or requiring intubation; given that their mortality is more likely to be determined by the severity of their clinical presentation rather than the PCI strategy. The primary endpoint was all-cause mortality. Survival data were obtained through the UK Health and Social Care Information Service. Clinical and angiographic data were collected from our prospectively collated database and supplemented from electronic records where required. All angiograms were reviewed by an experienced operator to confirm treatment strategy, bifurcation lesions, coronary artery dissection and TIMI flow pre- and post-intervention.

Results A total of 1190 patients were identified; 470 treated with DCB and 720 with DES. The average age for the DCB group was $65.5 \pm 12.5$ years old $(73.6 \%$ male); while for the DES group it was $65.5 \pm 11.6$ years old $(74.7 \%$ males). The average follow up was $882 \pm 439$ days and $978 \pm 426$ days for the DCB and DES group respectively. There were a few differences between the groups (table 1).
Abstract 48 Table 1

\begin{tabular}{|l|l|l|l|}
\hline & DCB (470) & DES (720) & P value \\
\hline HTN & $192(40.9 \%)$ & $251(34.9 \%)$ & 0.037 \\
\hline Smoking & $264(56.2 \%)$ & $455(63.2 \%)$ & 0.006 \\
\hline Bifurcations & $196(41.7 \%)$ & $213(29.6 \%)$ & $<0.001$ \\
\hline True bifurcations & $49(10.4 \%)$ & $40(5.6 \%)$ & 0.002 \\
\hline Vessel diameter & $3.28(0.54)$ & $3.52(0.6)$ & $<0.001$ \\
\hline
\end{tabular}

Abstract 48 Table 2

\begin{tabular}{|l|l|l|}
\hline & Hazard ratio (95\% CI) & P value \\
\hline Age & $1.081(1.058,1.106)$ & $<0.001$ \\
\hline Stroke & $2.453(1.148,5.243)$ & 0.021 \\
\hline Coronary artery bypass graft & $4.776(1.863,12.241)$ & 0.001 \\
\hline Heart failure & $5.121(1.314,19.964)$ & 0.019 \\
\hline COPD & $2.489(1.266,4.895)$ & 0.008 \\
\hline Diabetes & $1.831(1.063,3.153)$ & 0.029 \\
\hline
\end{tabular}

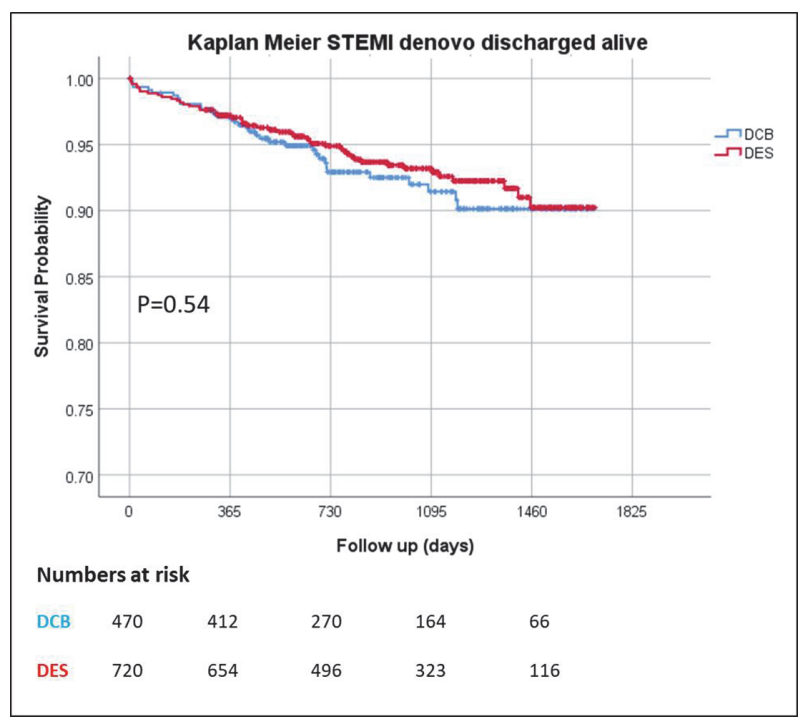

Abstract 48 Figure 1

There were no differences in all other clinical and angiographic characteristics. The all-cause mortality was $33 / 470$ (7\%) for the DCB group and 48/720 (6.7\%) for the DES group. Kaplan Meier estimator plot for all-cause mortality (figure 1) did not show a significant difference between DCB and DES $(p=0.54)$. On multivariable Cox regression analysis (table 2), age, prior stroke, coronary artery bypass graft, heart failure, chronic obstructive pulmonary disease (COPD) and diabetes were significant poor predictors of mortality.

Conclusion Our real-world data from a large, contemporary cohort of STEMI patients demonstrate that DCB only angioplasty is safe compared to DES and may be considered as a treatment option.

Conflict of Interest none 